\title{
Producción de sueros y vacunas en Chile, la importancia del abastecimiento local
}

\author{
Production of vaccines in Chile, the importance of local supply
}

\author{
Cecilia Ibarra M. ${ }^{1}$ y Mirtha Parada V. $^{2}$
}

${ }^{1}$ Centro de Ciencias del Clima y la Resiliencia, (CR), Universidad de Chile.

${ }^{2}$ Instituto de Salud Pública (ISP).

Financiamiento: la dedicación de la autora principal contó con el financiamiento del proyecto FONDAP / CONICYT 15110009

Declaramos no tener conflictos de interés.

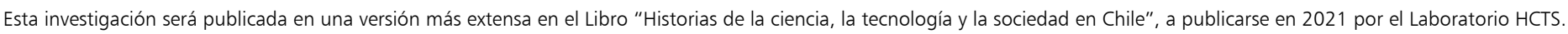

Recibido: 1 de junio de 2020 / Aceptado: 2 de julio de 2020

\section{Resumen}

En Chile, se produjeron vacunas desde fines del siglo XIX hasta inicios del siglo XXI de manera ininterrumpida. La producción se realizó en institutos del Estado, desde donde se abastecía al Servicio de Salud para los programas de vacunación. Además de fabricar, envasar y distribuir vacunas, los institutos del Estado, en particular el Instituto Bacteriológico (IB), exportaron vacunas a Latinoamérica y realizaron investigación y desarrollo, destacándose en este ámbito con la vacuna Fuenzalida-Palacios para el control de la rabia humana y canina. El presente estudio provee un listado de vacunas producidas en Chile en los institutos del Estado entre fines del siglo XIX y el año 2005, y una discusión sobre las visiones del rol de Estado que justificaron las acciones respecto de la producción de vacunas en Chile. El Estado desarrollista de mediados del siglo XX, dio paso al Estado neoliberal que se institucionalizó con la constitución de 1980 definiendo el rol subsidiario que ha guiado la discusión en materia de producción de vacunas locales en las últimas décadas.

Palabras clave: vacunas; producción farmacológica; política pública; Instituto Bacteriológico de Chile; historia de la ciencia y la tecnología en Chile.

\section{Introducción}

$\mathrm{E}$ n la última década se ha renovado el interés por la producción de vacunas en Chile, más aún en el contexto de la pandemia mundial de SARS-CoV-2, que mantiene a Chile en un estado de alerta sanitaria al igual que el resto del mundo. Por una parte,

\begin{abstract}
In Chile, vaccines were produced from the late 19th century to the early 21 st century without interruption. The production was carried out in State institutes, from where the health service was supplied for the vaccination programs. In addition to manufacturing, packaging and distributing vaccines, the State institutes, in particular the Instituto Bacteriológico (IB), exported vaccines to Latin America and carried out research and development, standing out in this area with the Fuenzalida-Palacios vaccine for the control of human and canine rabies. The present study provides a list of vaccines produced in Chile at State institutes between the end of the 19th century and 2005, and a discussion on the visions of the State role that justified the actions regarding the production of vaccines in Chile. The developmental State of the mid-twentieth century gave way to the neoliberal State that was institutionalized with the 1980 constitution, which defines the subsidiary role of the State that has guided the discussion on local vaccine production in the last decades.

Keywords: vaccines production; public policy; Instituto Bacteriológico de Chile; history of science and technology in Chile.
\end{abstract}

investigadores chilenos anuncian sus avances en la creación de una vacuna nacional a escala de laboratorio $\mathrm{y}$, por otra, se plantea la necesidad de producción local ante el temor de quedar sin acceso a la oferta internacional, considerando que los laboratorios priorizarán el abastecimiento en sus propios países. Chile ha vivido situaciones de des-abastecimiento de medicamentos importados durante la segunda 
guerra mundial ${ }^{1} \mathrm{y}$ ha enfrentado situaciones de crisis como los grandes terremotos de 1939 y 1960, donde fueron indispensables los sueros y medicamentos. En estos casos el Instituto Bacteriológico (IB) cumplió un rol fundamental en la producción y provisión de insumos médicos, en particular de sueros y vacunas.

Muchas de las vacunas básicas para los programas de vacunación masiva se produjeron localmente, no sólo en Chile, sino también en otros países latinoamericanos ${ }^{2}$, en algunos de los cuales aún se continúa con la producción local. La historia de la fabricación de vacunas chilenas está escasamente documentada. Las investigaciones disponibles se refieren de manera preferente a la historia de la vacunación y sólo algunos profundizan en vacunas específicas, que constituyeron hitos en el manejo de enfermedades infecciosas, como, por ejemplo, la vacuna de la viruela ${ }^{3}$, la historia de la rabia en $\mathrm{Chile}^{4}$ o la de la escarlatina $^{5}$, para la que se produjo vacuna antiescarlatinosa y suero anti-S. pyogenes.

La producción de vacunas en Chile se estableció, aumentando y diversificándose, con la creación de institutos del Estado que tenían entre sus funciones abastecer a los programas de vacunación. Aunque, existen algunos antecedentes de producción de sueros y vacunas en otras instituciones del Estado como el Instituto de Vacuna Animal Julio Besnard (IVA-JB) y el Instituto de Higiene (IH), este trabajo se centra en la producción nacional entre 1929, cuando se crea el Instituto Bacteriológico (IB), y 2005, año que establecimos como fecha aproximada del cierre de la producción en el ISP.

\section{Listado de vacunas y sueros producidos en Chile (1887-2005)}

La primera tarea de esta investigación fue construir un registro completo de producción de vacunas en Chile a partir de diversas fuentes. El objetivo fue cubrir la producción a partir de la creación del Instituto de Higiene (IH), luego en el Instituto Bacteriológico (IB), hasta el cierre en el Instituto de Salud Pública (ISP). A partir de esta compilación, resumida en la Tabla 1 , se constata que la producción fue sostenida en el tiempo. El IH creado en el año 1892, se inició en el área dando continuidad al trabajo del IVA-JB. La fecha de cierre de la producción se estableció en el 2005, aunque no hay un documento oficial que lo acredite. El área de producción del ISP fue cerrada en 2002; no obstante, quedó stock que se siguió envasando al menos hasta 2004. Los registros sanitarios como medicamentos de las vacunas caducaron en 2005 y no se renovaron, lo que se consideró como un hito de término de la producción.

La construcción del listado de vacunas se basó en una amplia revisión bibliográfica, cubriendo publicaciones científicas, reportes, instrumentos regulatorios y comunicados de las instituciones de salud (Ministerio de Salud, Organización Mundial de la Salud y sus divisiones para América Latina, IB, ISP). La revisión incluyó además material de archivo del IB y el ISP, como actas de Directorio, memorándums, oficios, faxes internos del área de producción, boletines, presentaciones en seminarios, reportes internos, libros de producción, reportes de evaluación y archivo de envases usados en el área producción. Además, se realizaron seis entrevistas a profesionales relacionados con la producción de vacunas en el IB y el ISP. Las fuentes asociadas a la identificación de cada vacuna se resumen en la Tabla 2.

\section{El rol del Estado en la producción de vacunas}

La vacunación comenzó en Chile en el siglo XIX. En esa época, el rol del Estado en la salud pública era entendido como un asunto de coordinación y apoyo a las acciones de beneficencia. Al acercarse el siglo XX, el Estado fue tomando un rol más activo en el área, hasta que a partir de la década de 1920 emerge lo que se ha conocido como Estado asistencial, con una clara responsabilidad respecto de la salud de la población. El Estado asistencial interviene el modelo capitalista de desarrollo y aplica legislación social e incorporación de la clase trabajadora a un sistema nacional de atención de salud y de previsión social, con lo que alivia la presión del descontento social ${ }^{6}$. Este rol fue cambiando, particularmente con las reformas realizadas durante la dictadura militar en los años setenta y ochenta $y$, desde entonces, el paradigma predominante en el área ha sido el rol subsidiario del Estado, donde la salud se gestiona con criterios de mercado ${ }^{7}$.

Las vacunas se usan para prevenir enfermedades humanas desde hace cientos de años, hay antecedentes de su uso en China y Turquía, al menos desde el siglo IV a.C. ${ }^{8}$. La primera vacuna usada en Sudamérica fue la anti-variólica, que llegó a Montevideo en 1805 y de allí fue distribuida a Argentina, Chile y Perú9. Desde entonces comenzó localmente la propagación del fluido que se usaba como vacuna y las campañas de vacunación de la población.

En Chile, la inoculación con este fluido empezó en 1806 , siendo reforzada después del proceso de independencia con la creación de la Junta de la Vacuna ${ }^{10}$. Los gobiernos republicanos mantuvieron campañas de vacunación, que se institucionalizaron en 1887 con la ley de vacunación anti-variólica obligatoria y la creación del IVA-JB. El Estado fue tomando un rol más activo en materia de salubridad e higiene, lo que se vio reflejado entre otras acciones, en la creación del Instituto de Higiene (IH), que funcionó entre 1892 y 1924 y que contaba con 


\section{Vacunología}

\begin{tabular}{|c|c|c|c|}
\hline Sueros/vacunas & Departamento, Institución & Inicio producción & Término producción \\
\hline s. V. Anti-variólica & IVA-JB & 1887 & 1929 (IB fab. de V. en ternera) \\
\hline V. Anti-rábica (virus Lab. Pasteur) & IVA-JB & 1887 & 1938 (IB fab. V. anti-rábica fenicada) \\
\hline S. Tuberculina & Serotopía, IH & 1896 & Luego es usado como reactivo de diagnóstico \\
\hline S. Anti-diftérico (suero heterólogo) & Serotopía IH & 1899 (caballos) & 2005 (última producción ISP) \\
\hline S. Anti-escarlatinoso + & Depto. Inmunología, IB & 1929 & 1945 (IB por aparición de antibióticos) ${ }^{\#}$ \\
\hline S. Anti-tetánico & Depto. Inmunología, IB & 1929 & 2005 (última producción ISP) \\
\hline S. Anti-tífico & Depto. Inmunología, IB & 1929 & 2005 (última producción ISP) \\
\hline S. Anti-meningocócico & Depto. Inmunología, IB & 1929 & 1945 (IB por aparición de antibióticos)\# \\
\hline S. Anti-perfingens & Depto. Inmunología, IB & 1929 & 2005 (última producción ISP) \\
\hline S. Anti-histolítico & Depto. Inmunología, IB & 1929 & 1945 (IB por aparición de antibióticos)\# \\
\hline S. Anti-oedematiens & Depto. Inmunología, IB & 1929 & 1945 (IB por aparición de antibióticos)\# \\
\hline V. Anti-variólica glicerinada & Depto. Inmunología, IB & 1930 & 1960 (IB) \\
\hline V. Anti-variólica desecada & Depto. Inmunología, IB & 1929 & 1978 (IB erradicación de viruela) \\
\hline V. Anti-tuberculosa (BCG) & Depto. Inmunología, IB & 1953 & 2005 (última producción ISP) \\
\hline V. Anti-escarlatinosa & Depto. Inmunología, IB & 1929 & 1945 (IB por aparición de antibióticos)\# \\
\hline V. Anti-neumocócica & Depto. Inmunología, IB & 1929 & No está clara la fecha \\
\hline V. Anti-coqueluche (B. pertussis) & Depto. Inmunología, IB & 1929 & 2005 (última producción ISP)* \\
\hline V. Anatoxina escarlatinosa & Depto. Inmunología, IB & 1929 & 1945 (IB por aparición de antibióticos)" \\
\hline V. Anti-gonocócica & Depto. Inmunología, IB & 1929 & 1945 (IB por aparición de antibióticos)\# \\
\hline V. Anti-estafilocócica & Depto. Inmunología, IB & 1929 & 1945 (IB por aparición de antibióticos)\# \\
\hline V. Anti-gripal & Depto. Inmunología, IB & 1929 & No está claro (en 1949 aún existía) \\
\hline V. Anti-tífica preventiva (Salmonella) & Depto. Inmunología, IB & 1929 & 2005 (última producción ISP) \\
\hline V. Anti-tífica curativa (Salmonella) & Depto. Inmunología, IB & 1929 & 2005 (última producción ISP) \\
\hline V. Anti-virus antipiogeno & Depto. Inmunología, IB & 1929 & 1945 (IB por aparición de antibióticos)\# \\
\hline V. Anti-virus stafilocócico & Depto. Inmunología, IB & 1929 & 1945 (IB por aparición de antibióticos)\# \\
\hline V. Anatoxina diftérica (toxoide) & Depto. Inmunología, IB & 1930 & 2005 (última producción ISP) \\
\hline V. Anti-colibacilar (cepas de E. coli) & Depto. Inmunología, IB & 1930 & 1945 (IB por aparición de antibióticos)\# \\
\hline V. Anti-ducrey (Haemophilus ducreyi) & Depto. Inmunología, IB & 1930 & 1945 (IB por aparición de antibióticos)" \\
\hline V. Anti-estreptocócica & Depto. Inmunología, IB & 1930 & 1945 (IB por aparición de antibióticos) ${ }^{\#}$ \\
\hline V. Anti-piógena polivalente (S. pyogenes) & Depto. Inmunología, IB & 1930 & 1945 (IB por aparición de antibióticos) \\
\hline V. Anti-pestosa (Yersinia pestis) & Depto. Inmunología, IB & 1930 & 1945 (IB por aparición de antibióticos)" \\
\hline V. Anti-tífica vía bucal & Depto. Inmunología, IB & 1930 & No está clara la fecha \\
\hline V. Anti-rábica fenicada & Sección antirrábica, IB & 1938 & 1954 (IB V. Fuenzalida Palacios) \\
\hline V. DP (vacuna mixta) & Sección vacunas, IB & 1943 & Década de 1970 (IB uso triple) \\
\hline V. Anti-rábica uso humano & Sección antirrábica, IB & $1959-60$ & 2005 (última producción ISP) \\
\hline V. Anti-rábica uso veterinario & Sección antirrábica IB & $1954-55$ & 2005 (última producción ISP) \\
\hline V. DT adulto & Sección vacunas, IB & Década de 1960 & 2005 (última producción ISP) \\
\hline V. DT infantil & Sección vacunas, IB & Década de 1960 & 2005 (última producción ISP) \\
\hline V. DPT & Sección vacunas, IB & Década de 1960 & 2005 (última producción ISP) \\
\hline
\end{tabular}




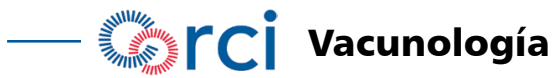

\begin{tabular}{|c|c|c|}
\hline Sueros/Vacunas & Institución, Fechas & Fuentes \\
\hline S. V. Anti-variólica & IVA-JB, 1887-1929 & Borgoño D, J. M. Vacunación antivariólica en Chile. Rev Chilena Infectol 2002; 19, 60-2; Acta No14, 1929 \\
\hline V. Anti-rábica (virus Lab. Pasteur) & IVA-JB, 1887-1938 & $\begin{array}{l}\text { Laval, E., \& Lepe, P. Una visión histórica de la rabia en Chile. Rev Chilena Infectol 2008; 25(2): S2-S7. } \\
\text { Acta No14, 1929; Acta N¹, 1936; Acta N²0, } 1938\end{array}$ \\
\hline S. Tuberculina & $\mathrm{H}, 1896-$ & Memoria del ISP, 1892-2009'; envases; Acta N¹, 1936 \\
\hline S. Anti-diftérico (suero heterólogo) & $\mathrm{IH}, 1899-2005$ & $\begin{array}{l}\text { Memoria del ISP, 1892-2009, pg 52; envases; Acta No1-No9, 1936; Acta 19, 1938; Acta No63-No65, } \\
1941 .\end{array}$ \\
\hline S. Anti-escarlatinoso & IB, 1929-1945 & Memoria del ISP, 1892-2009, pg 52; envases; Acta No1, 1936 \\
\hline S. Anti-tetánico & IB, 1929-2005 & $\begin{array}{l}\text { Memoria del ISP, 1892-2009, pg 52; envases; Acta Nº1- Nº, 1936; testimonio profesional que hizo } \\
\text { pruebas de potencia al suero desde los años 1970s; testimonios jefes de producción que van de 1970s } \\
\text { al cierre. }\end{array}$ \\
\hline S. Anti-tífico & IB, 1929-2005 & $\begin{array}{l}\text { Memoria del ISP, 1892-2009, pg 52; envases; Acta N¹-Nº9, 1936; Acta 17, 1937; testimonios jefes } \\
\text { de producción que van de 1970s al cierre. }\end{array}$ \\
\hline S. Anti-menigocócico & IB, 1929-1945 & Memoria del ISP, 1892-2009, pg 52; envases; Acta No1, 1936; Acta № 42, 1939 \\
\hline S. Anti-perfingens & IB, 1929-2005 & Memoria del ISP, 1892-2009, pg 52; envases; Acta No1, 1936 \\
\hline S. Anti-histolítico & IB, 1929-2005 & Memoria del ISP, 1892-2009, pg 52; envases; Acta №1, 1936 \\
\hline S. Anti-oedematiens & IB, 1929-1945 & Memoria del ISP, 1892-2009, pg 52; Acta N¹, 1936 \\
\hline V. Anti-variólica glicerinada & IB, 1930-1960 & $\begin{array}{l}\text { Borgoño D, J. M. Vacunación antivariólica en Chile. Rev Chilena Infectol, 2002; 19: 60-2; Acta No1-No9, } \\
\text { 1936; Acta No20, 1938; Acta No45, } 1940 .\end{array}$ \\
\hline V. Anti-variólica desecada & IB, 1940-1978 & $\begin{array}{l}\text { Borgoño D, J. M. Vacunación antivariólica en Chile. Rev Chilena Infectol 2002; 19: 60-2; envases; Acta } \\
\text { No1, 1936; Acta Na51, } 1940\end{array}$ \\
\hline V. Anti-tuberculosa (BCG) & IB, $1953-2005$ & $\begin{array}{l}\text { Actas Na } 158 \text { y } 161 \text { de 1952, Acta No163, 1953, Actas Nº1 a 3, 1960. Envases, testimonios jefes de } \\
\text { producción que van de 1970s al cierre. }\end{array}$ \\
\hline V. Anti-escarlatinosa & IB, 1929-1945 & $\begin{array}{l}\text { Memoria del ISP, 1892-2009, pg 52; Laval, E. La epidemia de escarlatina del año } 1929 \text { en Chile. Rev } \\
\text { Chilena Infectol 2009; 26(2): 168-72; envases; Acta No1, } 1936\end{array}$ \\
\hline V. Anti-neumocócica & IB, 1929-? & Memoria del ISP, 1892-2009, pg 52; Acta N¹, 1936 \\
\hline V. Anti-coqueluche (B. pertussis) & IB, 1929-2005 & $\begin{array}{l}\text { Memoria del ISP, 1892-2009, pg 52; Acta No1-No9, 1936; testimonio de profesional sobre trabajo en } \\
\text { control de calidad de esta vacuna en los 1970s }\end{array}$ \\
\hline V. Anatoxina escarlatinosa & IB, 1929-1945 & Memoria del ISP, 1892-2009, pg 52; Acta №1, 1936 \\
\hline V. Anti-gonocócica & IB, 1929-1945 & Memoria del ISP, 1892-2009, pg 52; Acta N¹, 1936 \\
\hline V. Anti-estafilocócica & IB, 1929-1945 & Memoria del ISP, 1892-2009, pg 52; Acta N¹, 1936 \\
\hline V. Anti-gripal & IB, 1929-1949 & Memoria del ISP, 1892-2009, pg 52; envases; Acta N²21, 1929; Acta Nº1, 1936 \\
\hline V. Anti-tífica preventiva (Salmonella) & IB, 1929-2005 & $\begin{array}{l}\text { Memoria del ISP, 1892-2009, pg 52; envases; Acta N¹, 1936; testimonios jefes de producción que } \\
\text { van de 1970s al cierre; planilla de producción 2001, plan de acción área producción 2004, fax y oficios } \\
\text { 2001-2003 entre departamento de producción y dirección ISP (archivo personal entrevistado) }\end{array}$ \\
\hline V. Anti-tífica curativa (Salmonella) & IB, 1929-2005 & $\begin{array}{l}\text { Memoria del ISP, 1892-2009, pg 52; envases; Acta N¹, 1936; testimonios jefes de producción que } \\
\text { van de 1970s al cierre; planilla de producción 2001, plan de acción área producción 2004, fax y oficios } \\
\text { 2001-2003 entre departamento de producción y dirección ISP (archivo personal entrevistado) }\end{array}$ \\
\hline V. Antivirus antipiogeno & IB, 1929-1945 & Memoria del ISP, 1892-2009, pg 52; Acta N¹, 1936 \\
\hline V. Antivirus stafilocócico & IB, 1929-1945 & Memoria del ISP, 1892-2009, pg 52; Acta N¹, 1936 \\
\hline V. Anatoxina diftérica (toxoide) & IB, 1930-2005 & $\begin{array}{l}\text { Memoria del ISP, 1892-2009, pg 53; envases; Acta N¹, 1936, testimonios jefes de producción que } \\
\text { van de 1970s al cierre. }\end{array}$ \\
\hline V. Anti-colibacilar (cepas de E. coli) & IB, 1930-1945 & Memoria del ISP, 1892-2009, pg 53; Acta N¹, 1936 \\
\hline V. Anti-ducrey (Haemophilus ducreyi) & IB, 1930-1945 & Memoria del ISP, 1892-2009, pg 53; Acta N¹, 1936 \\
\hline V. Anti-estreptocócica & IB, 1930-1945 & Memoria del ISP, 1892-2009, pg 53; Acta N¹, 1936 \\
\hline V. Anti-piogena polivalente (S. pyogenes) & IB, 1930-1945 & Memoria del ISP, 1892-2009, pg 53; Acta N¹, 1936 \\
\hline V. Anti-pestosa (Yersinia pestis) & IB, 1930-1945 & Memoria del ISP, 1892-2009, pg 53; Acta No1, 1936 \\
\hline
\end{tabular}




\section{IB, 1930-?}

IB, 1938-1954

IB, 1943-1970s

IB, 1959/60-2005

V. Anti-rábica uso humano

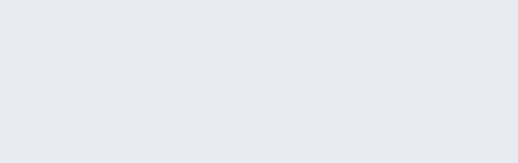

V. Anti-rábica uso veterinario

IB, 1954/55-2005

V. DT adulto

V. DT infantil

V. DPT
IB, 1960s-2005

IB, 1960s-2005

IB, 1960s-2005
Memoria del ISP, 1892-2009, pg 53; Acta N¹, 1936

Laval, E., \& Lepe, P. Una visión histórica de la rabia en Chile. Rev Chilena Infectol 2008: 25(2): S2-S7, Acta N²4, 1940; Acta N059, 1941

\section{Historia del Instituto de Salud Pública de Chile, camino al bicentenario, página 57; envases}

Álvarez, J. P. Eduardo Fuenzalida Loyola y su contribución a la lucha contra un enemigo mortal: la familia de los Rhabdoviridae, género Lyssavirus. Revista Médica Clínica Las Condes 2015; 26(6): 8138.); Envases; entrevista profesional 1968-..., testimonio profesional que hizo pruebas de potencia al suero desde los años 1970s; testimonios jefes de producción que van de 1970s al cierre; planilla de producción 2001, plan de acción área producción 2004, fax y oficios 2001-2003 entre departamento de producción y dirección ISP (archivo personal entrevistado)

Álvarez, J. P. (2015). Eduardo Fuenzalida loyola y su contribución a la lucha contra un enemigo mortal: la familia de los Rhabdoviridae, género Lyssavirus. Revista Médica Clínica Las Condes 2015; 26(6), 813-8. Envases; testimonio profesional que hizo pruebas de potencia al suero desde los años 1970s; testimonios jefes de producción que van de 1970s al cierre; planilla de producción 2001, plan de acción área producción 2004, fax y oficios 2001-2003 entre departamento de producción y dirección ISP (archivo personal entrevistado)

Envases, testimonios jefes de producción que van de 1970s al cierre; planilla de producción 2001, plan de acción área producción 2004, fax y oficios 2001-2003 entre departamento de producción y dirección ISP (archivo personal entrevistado)

Envases, testimonios jefes de producción que van de 1970s al cierre. Registro de Fax del área producción años 2000s; planilla de producción 2001, plan de acción área producción 2004, fax y oficios 2001-2003 entre departamento de producción y dirección ISP (archivo personal entrevistado)

Envases; testimonio profesional que hizo pruebas de potencia al suero desde los años 1970s, testimonios jefes de producción que van de 1970s al cierre; planilla de producción 2001, plan de acción área producción 2004, fax y oficios 2001-2003 entre departamento de producción y dirección ISP (archivo personal entrevistado)

Fuente: Elaboración propia. Abreviaturas: S: suero, V: vacuna, IH: Instituto de Higiene, IB: Instituto Bacteriológico, ISP: Instituto de Salud Pública, BCG: Bacillus de Calmette y Guérin, IVA-JB: Instituto de Vacuna Animal Julio Besnard, DT: difteria tétanos, DPT: difteria, tétanos y pertussis (coqueluche). 'La información original para todas las vacunas que reporta la Memoria del ISP proviene de la Memoria del Instituto Bacteriológico de Chile de 1929 y la Revista del Instituto Bacteriológico de Chile, Vol. I ( marzo) 1930, que también revisamos.

un área de serotopía (hoy serología). El modelo prevalente en el siglo XIX fue el de la beneficencia, de un Estado caritativo que se ocupaba de la atención de salud apoyado por organizaciones de beneficencia ${ }^{11}$.

La relevancia de los asuntos sanitarios se agudizó a principios del siglo XX. Eventos como la "gripe española" de 1918 alimentaron discusiones sobre el rol del Estado en la prevención y manejo de la pandemia y la importancia de la medicina preventiva ${ }^{12}$. La crisis sanitaria era profunda y fue un factor importante en la emergencia de la "cuestión social". Se necesitaba de un pueblo sano para lograr el desarrollo económico ${ }^{13}$, la salud hermanaba las preocupaciones sociales y económicas, y el rol de Estado en la salud pública parecía preponderante ${ }^{14}$. Estas ideas promovieron reformas en el sector y la reorganización de los servicios sanitarios del país ${ }^{15}$.

En este contexto se crea el Instituto Bacteriológico (IB), que fue el heredero del IH. La ley de creación del IB - Ley 4.557 de 1929- decretó que su misión sería: la formación de bacteriólogos para el país, la elaboración de sueros, vacunas, productos biológicos y bioquímicos, y, además, el control de la fabricación y venta de estos productos en el país ${ }^{16}$. El IB debía entregar estos productos al gobierno para la atención de salud a precio de costo ${ }^{17}$.

La crisis sanitaria se mantenía aguda; en la década de 1930, Chile experimentaba epidemias de viruela, cólera y de tifus exantemático ${ }^{18}$, con altas tasas de mortalidad. Los problemas sanitarios se magnificaban con la migración hacia las urbes desde los centros mineros abandonados y las zonas rurales pobres. El hacinamiento en las ciudades aumentaba y, además, se empezaban a vivir los efectos económicos de la segunda guerra mundial ${ }^{19}$.

La producción de sueros y vacunas aumentó con la creación del IB. Este instituto contaba con seis áreas en las que se hacía investigación y diagnóstico de enfermedades, se producían y envasaban algunos preparados farmacológicos. En el Departamento de Inmunología se elaboraban los sueros y vacunas para la prevención y tratamiento de enfermedades infecciosas ${ }^{20}$. 
En las actas del Consejo del IB están descritos los informes al Directorio que daban cuenta del seguimiento de las actividades de producción, investigación y desarrollo. En materia de sueros y vacunas hay referencias a mejoras permanentes de técnicas, aumentos de productividad y nuevos desarrollos. Entre las estrategias relacionadas al rol del IB en la gestión de eventuales crisis sanitarias, se documenta la mantención de stocks de seguridad de sueros en casos de epidemias o cataclismos. Esta estrategia resultaba anti-económica para el IB, pero era considerada como un deber, que probó sus beneficios durante emergencias como el terremoto del año $1939^{21}$.

Un logro destacado del IB fue la creación, desarrollo y producción de la vacuna Fuenzalida-Palacios para el enfrentamiento de la rabia humana y animal. Este es uno de los pocos desarrollos tecnológicos del IB que puede considerarse conocido y sobre los que se ha escrito ${ }^{22}$. La vacuna Fuenzalida-Palacios obtuvo reconocimiento internacional y sus creadores recorrieron diversos países enseñando a producirla, sin nunca patentarla.

En América Latina, no sólo el IB producía sueros y vacunas, también lo hacían al menos el Instituto Bacteriológico Carlos Malbrán en Argentina y el Instituto Butantan en Brasil ${ }^{23}$. Todos estos institutos estatales mantenían vínculos de colaboración entre ellos, tanto en investigación, desarrollo y capacitación, como en comercialización de productos.

La década de los cincuenta, cuando se unificó la atención de salud en el Servicio Nacional de Salud (SNS) tuvo sus tensiones y situaciones de crisis en cuanto al financiamiento de la salud, la saturación de los servicios y por asuntos institucionales de organización y dependencia del $\mathrm{SNS}^{24}$. Los valores sociales de la salud pública se fueron tensando entre dos posturas opuestas; por una parte, las visiones económicas neoliberales, que priorizaban la rentabilidad económica de las prestaciones de salud y proclamaban la eficiencia de un sistema de atención privatizado y, por otra, las ideas de la medicina social, y más tarde el surgimiento del proyecto de transformación que llevó a la Unidad Popular al gobierno en $1970^{25}$.

A partir del golpe de Estado el 11 de septiembre de 1973, fueron ganado terreno las ideas neoliberales respecto del rol del Estado. La dinámica de la oferta y demanda de salud debía ser regulada por el Estado. En la ideología dominante que se fue instalando de manera hegemónica desde entonces, la intervención estatal sólo se justifica cuando hay fallas de mercado que los privados no pueden solucionar por si solos. Como estableció la Constitución de la República de 1980, el rol del Estado es subsidiario al rol del mercado. El Estado debe garantizar el libre acceso a la salud, la coordinación y el control de la ejecución de prestaciones de salud en instituciones públicas o privadas ${ }^{26}$.
Apenas cambió la administración en 1973, se suspendió toda inversión en equipamiento en vacunas, otras actividades productivas fueron cerradas o trasladadas, como la producción de penicilina ${ }^{27}$, cloranfenicol, éter, insulina y hormonas tiroideas. La producción de vacunas se mantuvo con un presupuesto mínimo para operación, lo que puso presión sobre el equipo profesional para mantener los estándares de calidad internacionales. Mientras, la producción de vacunas en el mundo fue desarrollando nuevas tecnologías, incorporando, por ejemplo, la automatización de procesos.

Luego vino el proceso de reformas concordantes con el rol subsidiario de un Estado eficiente y pequeño, en que el IB pasó a ser el Instituto de Salud Pública (ISP) por Decreto Ley 2763, de $1979^{28}$. La creación del ISP consolida un rol preferentemente regulador, al que le corresponde servir de laboratorio nacional de referencia, normalizador y supervisor; ejercer acciones de control de calidad de medicamentos y otros insumos médicos; ser el organismo productor del Estado, elaborando productos en casos calificados y con autorización del Ministerio de Salud; prestar servicios de asesoría, investigación, capacitación y fiscalización ${ }^{29}$. El Estado debe garantizar el libre acceso a la salud, la coordinación, el control y la ejecución de las prestaciones de salud en instituciones públicas o privadas ${ }^{30}$. El ISP puede producir insumos médicos, pero ésta es una función secundaria frente al rol de ente normalizador y supervisor, y que debe justificarse y obtener aprobación del Ministerio de Salud ${ }^{31}$. El rol productor del ISP ha sido desde entonces objetivo de discusiones internas, reportadas en las entrevistas ${ }^{32}$, donde el discurso oficial y dominante argumenta a favor del rol subsidiario, la imposibilidad de ser "juez y parte" al producir y regular, y la supuesta idoneidad del sector privado en la actividad productiva. Esta ideología ha tenido como consecuencia el abandono de las actividades productivas del ISP en sueros, vacunas y medicamentos.

Las visiones respecto de la producción del ISP no cambiaron con la recuperación de la democracia en 1990. Los entrevistados recuerdan los años noventa como un período de presión de parte de los laboratorios privados para la desaparición de la producción del ISP. A principios de los noventa hubo un intento por reforzar la producción de vacunas y se creó una unidad de investigación y desarrollo, donde se contrataron nuevos investigadores calificados. Esta unidad tuvo proyectos para desarrollar la vacuna contra Haemophilus influenza tipo b y una vacuna para proteger la producción acuícola de salmones. No obstante, el interés demostrado en esta primera etapa de la administración democrática se fue perdiendo con sucesivos cambios de Dirección del ISP y no se realizaron inversiones en el área ni se priorizó en su presupuesto.

A inicios del nuevo siglo, los profesionales del ISP temían un inminente cierre de la producción de vacunas, 
Correa, con el objetivo de que exista una institución especializada en investigación científica, que genere y difunda conocimientos científicos y tecnológicos en salud, además de la prestación de servicios de laboratorio especializado en el ámbito de la salud pública.

Los argumentos de la OPS para una producción estatal de vacunas básicas para los programas nacionales de vacunación son de carácter estratégico: garantizar la protección de la salud de la población, regular los precios, mantener independencia de los países proveedores, responder de manera oportuna frente a enfermedades emergentes, usar cepas autóctonas, proveer seguridad estratégica frente a catástrofes y conflictos, promover la investigación y el desarrollo nacional, cubrir la demanda no satisfecha de biológicos a nivel mundial y producir vacunas huérfanas o no tradicionales. El "Comité de defensa de las vacunas del ISP” esgrimió también estas razones.

La evaluación económica, deja fuera aspectos estratégicos. Invertir en la actualización tecnológica de la planta de producción de vacunas necesitaba de voluntad política, basada en razones estratégicas de largo plazo. Los países de la región han tomado decisiones políticas distintas. Argentina, por ejemplo, a partir de la crisis de 2001 decidió que la salud de la población debía ponerse por sobre otros intereses y que las vacunas y otros medicamentos serían tratados como un bien público, a lo que siguió una política de reimpulso de la producción estata ${ }^{39}$.

El discurso hegemónico del rol subsidiario del Estado llevó a la des-inversión en la producción de vacunas en el ISP. No invertir es una decisión de política pública, que tiene un impacto en el largo plazo en cualquier área, más aún en un área con gran dinamismo en la innovación tecnológica. Esta decisión permitió terminar con la producción evitando el potencial conflicto de abrir una discusión democrática respecto del cierre. La obsolescencia aparece como la culpable, sin reconocer que es el resultado de las decisiones de planificación y presupuesto de cada administración, lo que, en estos días, en la política climática, se ha llamado "el costo de la inacción". Este costo deja oculta la responsabilidad de la inacción e impide el debate democrático porque nada hay que debatir cuando ya no hay planta competitiva.

La discusión política sobre la producción estatal no se ha dado, a pesar de que en este siglo el país ha vivido escasez de vacunas de sarampión para los programas de vacunación obligatoria por falta de oferta internacional, alarmas por guerra biológica y problemas de abastecimiento relativos a la calidad de las vacunas importadas y su alto valor comercial. Chile depende totalmente de las importaciones que han probado tener costos altos e incertidumbre en el abastecimiento. Además, en el caso de las vacunas estacionales, como la vacuna de la influenza, Chile no cuenta con vacunas elaboradas con cepas locales, que pudieran conferir mayor cobertura 
inmunitaria a la población. No hay producción nacional privada y las condiciones de mercado hacen altamente improbable que exista y el país no tiene stock de seguridad de suministros básicos.

\section{Comentario final}

Esta investigación partió con la construcción del listado de vacunas producidas en Chile en los institutos del Estado. La producción fue sostenida en el tiempo, con talento y capacidades locales. La innovación tecnológica que significó producir por primera vez cada nueva vacuna en Chile vino seguida de mejoras e innovaciones incrementales de proceso y la solución de desafíos para seguir produciendo en condiciones de desinversión, en que, a pesar de la precariedad tecnológica, se mantuvo el control de la calidad de los productos. No ha faltado talento y se ha mantenido la visión compartida de la importancia de las vacunas para la salud de la población, se mantienen las campañas de vacunación y la población, en general, responde y valora estas acciones. Está claro que lo que ha faltado es una discusión abierta que no asuma como dogma la imposibilidad que el Estado produzca medicamentos como política estratégica.
La pandemia de SARS-CoV-2 ofrece quizá una oportunidad para repensar la estrategia de abastecimiento de vacunas y el rol de la producción estatal. Sobre todo, considerando que, con esta emergencia sanitaria global, hemos podido comprobar la importancia de que cada país cuente con producción local de todo tipo de productos como insumos médicos, reactivos químicos, materias primas para alimentos, etc., en que los medicamentos y las vacunas juegan un rol primordial. Esperamos que este artículo pueda aportar con una mirada histórica a una discusión democrática con visión de largo plazo.

Agradecimientos: Este trabajo contó con el apoyo del proyecto FONDAP/CONICYT 15110009 y con la oportunidad de discutir y difundir resultados en las instancias provistas por el Laboratorio de la Historia de la Ciencia la Tecnología y la Sociedad y la Red CTS-Chile. Las autoras agradecen la generosa contribución de los profesionales con trayectoria en el área vacunas del ISP: Ricardo González, Gastón Hernández, Luis Eduardo Johnson, Luis Rodríguez, Marcelo Sánchez y Abel Vásquez, y de la consultora en salud Patricia Zimermann, que entregaron sus testimonios y compartieron sus archivos personales.

\section{Referencias bibliográficas}

1.- Parada M. Salud Pública, medicamentos y pandemia. Cuadernos Médico Sociales: especial pandemia 2020; $1: 39-42$. https://cms. colegiomedico.cl/

2.- Homma A, Fabio J L di, de Quadros C. Los laboratorios públicos productores de vacunas: el nuevo paradigma. Rev Panam Salud Pública/Pan Am J Public Health 1998; 4 (4). https://iris.paho.org/ bitstream/handle/10665.2/8529/4n4a1. pdf?sequence $=1$ \&isAllowed $=y$

3.- Caffarena Barcenilla P. Salud pública, vacuna y prevención. La difusión de la vacuna antivariólica en Chile, 1805-1830. Historia 2016; 49 (2): 347-70. https://scielo.conicyt.cl/ pdf/historia/v49n2/art01.pdf

4.- Laval E, Lepe P. Una visión histórica de la rabia en Chile. Rev Chilena Infectol 2008; 25 (2): S2-S7. http://dx.doi.org/10.4067/S071610182008000200014

5.- Laval E. La epidemia de escarlatina del año 1929 en Chile. Rev Chilena Infectol 2009; 26(2): 168-72. http://dx.doi.org/10.4067/S071610182009000200010.

6.- Illanes M A. En el nombre del pueblo, del estado y de la ciencia: historia social de la salud pública Chile 1880/1973: hacia una historia social del siglo XX. Santiago de Chile: Ministerio de Salud, 2001.

7.- Molina C. Institucionalidad Sanitaria Chilena 1889-1989. Santiago de Chile: LOM Editores, 2010

8.- Iglesias-Gamarra A, Siachoque H, Pons-Estel B, Restrepo J F, Quintana G, Gómez Gutiérrez, A. La historia de la autoinmunidad. Primera Parte. La Inmunología, ¿desde dónde y hacia dónde? Revista Colombiana Reumatol 2009; 16(1): 11-31. http://www.scielo.org.co/pdf/rcre/ v16n1/v16n1a02.pdf

9.- Caffarena, op. cit.

10.- Caffarena, ibid.

11.- Illanes, op. cit. y Molina, op. cit.

12.- López M, Beltrán M. Chile entre pandemias: la influenza de 1918, globalización y la nueva medicina. Rev Chilena Infectol 2013. http://dx.doi.org/10.4067/S071610182013000200012.

13.- Araya C, Leyton C, López M, Palacios C, Sánchez M. República de la salud. Fundación y ruinas de un país sanitario Chile, siglos XIX y XX. Santiago de Chile, Ocho Libros, 2016.

14.- Illanes, op. cit.

15.- Illanes, ibid.

16.- Ley 4557 de 1929, Artículo $1^{\circ}$. Disponible en: https://www.leychile.cl/ Navegar?idNorma $=24756$.
17.- Ibid, Artículo $4^{\circ}$.

18.- Altamirano T C. "Desinfectorio Público", en Ferrer, P.L., Higiene y Asistencia Pública en Chile, Santiago, 1911; Corbalán Melgarejo, R., Nota de la Dirección General de Sanidad al Ministro del Interior, sobre los Informes de la Comisión designada por el Gobierno para estudiar los Servicios Sanitarios, Santiago, 1922; Medina C., Eduardo, Dr., y Toro A., Jorge, Dr. "Sinopsis Sanitaria Chilena", en Anales Chilenos de Historia de la Medicina; Vol. 17: 77-93, Santiago, 2007. Laval, E. (2013). Epidemia de tifus exantemático en Chile (1932-1939). 30 (3): 313-6.

19.- Illanes, op. cit.

20.- ISP (2009). Historia del Instituto de Salud Pública de Chile, 1892-2009. Ministerio de Salud, 2009: 44.

21.- Actas Directorio IB, 1929-1960.

22.- Laval E, Lepe P. op. cit; Álvarez, J. P. (2015). Eduardo Fuenzalida y su contribución a la lucha contra un enemigo mortal: la familia de los Rhabdoviridae, género Lyssavirus. Revista Médica Clínica Las Condes 2015; 26 (6): 813-8. https://www.elsevier.es/esrevista-revista-medica-clinica-las-condes-202pdf-S0716864015001595

23.- Ramacciotti K I, Romero L. Iniciativas 


\section{Vacunología}

estatales de producción y comercialización de medicamentos. Argentina, 1947- 2014.

En Y. Carvajal y M. J. Correa Gómez (eds.) Historia de los medicamentos. Santiago de Chile Ocho Libros, 2016.

24.- Illanes, op. cit.

25.- Molina, op. cit. e Illanes, ibid.

26.- Constitución de 1980, Capítulo III, Artículo $19^{\circ}$.

27.- Parada M, Ibarra C. Historia de la Producción de Penicilina en Chile (1943-1973). En Y. Carvajal y M. J. Correa Gómez (eds.). Historia de los medicamentos. Santiago de Chile Ocho Libros, 2016.
28.- Decreto Ley 2763,1979 . Reorganiza el Ministerio de Salud y crea Servicios de Salud, el Fondo Nacional de Salud, el Instituto de Salud Pública y la Central de Abastecimientos del Sistema Nacional de Servicios de Salud. Artículo $1^{\circ}$. Disponible en: https://www.leychile.cl/ Navegar?idNorma $=6999$.

29.- Decreto Ley $2763 \ldots$... Título $4^{\circ}$.

30.- Constitución de 1980, Capítulo III, Artículo $19^{\circ}$.

31.- Decreto 1222 de 1996. Aprueba Reglamento del Instituto de Salud Pública de Chile. Disponible en: https://www.leychile.cl/

Navegar?idNorma $=75287$.

32.- Tema coincidente en las entrevistas a los funcionarios y ex funcionarios del ISP.

33.- Homma, A., et al., op. cit.

34.- https://www.ins.gov.co/Paginas/Inicio.aspx

35.- http://www.inhrr.gob.ve/

36.- http://www.butantan.gov.br/

37.- https://www.birmex.gob.mx/inh.html

38.- http://www.investigacionsalud.gob.ec/ direccion-ejec/

39.- Ramacciotti, K. y Romero, L. La regulación de medicamentos en la Argentina (1946-2014). Revista CTS 2017; 12: 153-74. 